\title{
Plasma Neuropeptide Y (NPY) Increases in Humans in Response to the $\alpha_{2}$ Antagonist Yohimbine
}

Ann M. Rasmusson, M.D., Steven M. Southwick, M.D., Richard L. Hauger, M.D., and Dennis S. Charney, M.D.

Previous studies have shown that the intravenous administration of yohimbine, an $\alpha_{2}$ antagonist, increases norepinephrine turnover and has related anxiogenic effects in humans. We herein report that yohimbine also increases plasma neuropeptide $Y$ (NPY) in healthy human subjects. This finding is consistent with previous reports in animals, but contrasts with a previously reported study in humans. NPY is a 36 amino acid peptide neurotransmitter located in sympathetic and nonsympathetic nerve fibers, as well as in brain structures such as the locus coeruleus, where it is colocalized with norepinephrine. NPY has been shown to inhibit locus coeruleus neuronal firing, decrease norepinephrine release, and increase postsynaptic noradrenergic signal transduction. When administered centrally, NPY also has anxiolytic properties. This study therefore suggests that yohimbine challenge may be useful in assessing NPY and noradrenergic system interactions in neuropsychiatric disorders such as panic disorder or post traumatic stress disorder in which noradrenergic system dysfunction has been observed.

[Neuropsychopharmacology 19:95-98, 1998]

Published by Elsevier Science Inc.
KEY WORDS: Yohimbine; Neuropeptide Y (NPY); Human; Noradrenergic

Neuropeptide Y (NPY) is a 36 amino acid peptide neurotransmitter located in most sympathetic nerve fibers, as well as in nonadrenergic perivascular, enteric, cardiac nonsympathetic, and parasympathetic nerves (Wahlestedt and Reis 1993). NPY is also found in brain structures such as the amygdala, cortex, hippocampus, periaqueductal grey, and locus coeruleus, where it is co-localized with norepinephrine (Heilig and Widerlov 1990), a neurotransmitter thought to be involved in the neurobiology of stress and anxiety disorders. In the locus

From the Department of Psychiatry (AMR, SMS, DSC), Yale University School of Medicine, New Haven, CT; the National Center for PTSD (AMR, SMS, DSC), VA Connecticut, West Haven, CT; and the School of Medicine (RLH), University of California, San Diego, CA.

Address correspondence to: Ann M. Rasmusson, M.D., Psychiatry Service/116A, VA Connecticut, West Haven, 950 Campbell Avenue, West Haven, CT 06516. coeruleus, NPY potentiates $\alpha_{2}$-adrenoceptor-mediated inhibition of neuronal firing (Illes and Regenold 1990). NPY has also been shown to decrease the release of norepinephrine and enhance activation of postsynaptic neurons via receptor-mediated increases in $\mathrm{Ca}^{2+}$ conductance (Colmers and Bleakman 1994). Therefore, as sympathetically-derived NPY is preferentially released in response to high frequency stimulation (Pernow 1988), it appears that NPY functions to homeostatically regulate norepinephrine release, as well as increase the "synaptic gain" of noradrenergic neurotransmission.

Yohimbine is a noradrenergic $\alpha_{2}$ antagonist which induces anxiety while increasing plasma norepinephrine and 3-methyl-4-hydroxyphenylglycol (MHPG) in humans (Holmberg et al. 1962; Charney et al. 1982). Given that norepinephrine and NPY are co-localized and co-released from sympathetic neurons, we hypothesized that NPY levels would also increase in response to yohimbine in humans and that baseline NPY would negatively correlate with the peak MHPG response. 


\section{METHODS}

Eight healthy male subjects were included in the study (aged $27.9 \pm 9.5$ years; range: 21.1-43.7 years). They comprised a subset of control subjects from a previously reported study (Southwick et al. 1993). Subjects were administered normal saline or yohimbine $(0.4$ $\mathrm{mg} / \mathrm{kg}$ ) in a double-blind, random fashion, at 10:00 A.M. Blood samples were obtained at the following timepoints relative to injection: $-30,-15,+40,+60$, +120 , and +180 minutes. The first blood sample was obtained at least 60 minutes after placement of the intravenous catheter. Plasma was stored at $-70^{\circ} \mathrm{C}$ from the time of initial collection. NPY was measured after plasma extraction using a double antibody RIA using ${ }^{125}$ I-NPY as the tracer. This RIA possesses an assay sensitivity of $20 \mathrm{pg} / \mathrm{ml}$ and intra- and inter-assay coefficients of variation of $8 \%$ and $10 \%$, respectively (Allen et al. 1991). MHPG was measured by mass spectrometry as previously described (Southwick et al. 1993).

The average of the -30 and -15 minute samples was used as baseline for each condition. A two-way univariate repeated measures analysis of variance was used to assess the effect of condition (placebo vs. yohimbine) and time, as well as their interaction, on the level of plasma NPY. Planned contrasts were used to determine whether differences between the conditions were present at each time point. The peak change for plasma NPY following yohimbine or placebo was measured by subtracting the baseline level from the peak level. The peak change for yohimbine minus the peak change for placebo gave a net peak effect for yohimbine. Correlations between baseline NPY and the percent change in MHPG (measured between baseline and the peak MHPG level for each subject), and between the percent change in NPY and MHPG were assessed on the yohimbine challenge day only.

\section{RESULTS}

Plasma NPY was significantly increased after yohimbine, compared to placebo, at $+40,+60,+120$, and +180 minutes (Fig. 1). NPY peaked at 120 minutes after yohimbine injection for a net peak increase of $45.6 \%$, compared to placebo. Yohimbine also induced a $21.2 \%$ net increase in plasma MHPG. There was a positive correlation between the percent change in plasma NPY and



Figure 1. Plasma neuropeptide $\mathrm{Y}$ (NPY) in response to the intravenous injection of normal saline or yohimbine (0.4 mg/ $\mathrm{kg}$ ). There was a significant condition effect, $\mathrm{F}(1,7)=13.67, p<.008$, and a significant condition by time interaction effect, $\mathrm{F}(1,4)=4.53, p<.008$, on plasma NPY levels. Planned contrasts indicated that plasma NPY was significantly increased after yohimbine, compared to the placebo condition, at $+40,+60,+120$, and +180 minutes after injection: ${ }^{*} p<.05$; ${ }^{* * *} p<.001$; ${ }_{* * * *} p=.0001$. Each timepoint represents the mean \pm the standard error of the mean $(n=6-8)$. 
MHPG $(r=0.734, p<.04)$ and a trend toward a negative correlation between baseline NPY and the percent change in MHPG $(r=-0.685, p=.061)$ on the yohimbine challenge day.

\section{DISCUSSION}

Yohimbine previously has been used to elicit a hyperadrenergic anxiety state via antagonism of peripheral $\alpha_{2}$ noradrenergic autoreceptors (Holmberg et al. 1962; Charney et al. 1982; Goldberg and Robertson 1983; Henaur et al. 1984). Indeed, in the current study, administration of $0.4 \mathrm{mg} / \mathrm{kg}$ yohimbine induced a $21 \%$ increase in norepinephrine, as well as a $46 \%$ increase in NPY. These findings compare to the results of a previous study in humans by Hedner et al. (1992), in which plasma norepinephrine increased three-fold, while NPY did not significantly change in response to $0.25 \mathrm{mg} / \mathrm{kg}$ yohimbine. The discrepancy between studies may be due to differences in the sensitivity and specificity of the NPY radioimmunoassays used (Edvinsson et al. 1990; Allen et al. 1991); also, yohimbine-stimulated NPY release has been shown previously to be dose-dependent (Tavernier et al. 1992). The current findings also support studies demonstrating that plasma NPY is preferentially released in situations of high sympathetic nerve activity (Archeolos et al. 1987; Pernow and Lundberg 1989; Lundberg et al. 1989; Haass et al. 1989; Dahlof et al. 1991; Wahlestedt and Reis 1993), including vigorous exercise (Pernow 1988) and electroconvulsive therapy (Hauger et al. in preparation). The positive correlation between the percent change in NPY and MHPG in response to yohimbine is consistent with the corelease of these co-localized neurotransmitters from peripheral sympathetic neurons. The trend toward a negative correlation between baseline NPY and the percent change in MHPG in response to yohimbine is consistent with previous observations that NPY inhibits the release of MHPG from sympathetic neurons (Colmers and Bleakman 1994).

In a variety of animal models, centrally administered NPY has anxiolytic effects (Heilig et al. 1989, 1993; Wahlestedt et al. 1993). In humans, cerebrospinal fluid NPY levels have been found to correlate negatively with anxiety, but not depressive symptoms (Widerlov et al. 1989). This suggests that NPY may play a role in regulating anxiety, possibly via its effects on the noradrenergic system response to stress.

Previous studies have revealed greater peripheral noradrenergic system responses to yohimbine in patients with panic disorder and posttraumatic stress disorder (PTSD) compared to healthy controls (Charney et al. 1984; Gurguis and Uhde 1990; Charney et al. 1992; Southwick et al. 1993). This raises the possibility that differences in NPY levels or function might mediate or accompany these abnormalities. However, studies of plasma NPY levels in anxiety disorders have thus far yielded discrepant results. In one study, baseline plasma NPY was high in panic disorder patients compared to healthy controls (Boulenger et al. 1996), whereas another study reported baseline plasma NPY to be no different in patients with panic disorder or social phobia compared to healthy controls (Stein et al. 1996). However, given that NPY may play a homeostatic role in regulating the noradrenergic system, further investigation of NPY in the anxiety disorders is warranted and may benefit from a study design in which the noradrenergic system is activated, as for example, by yohimbine challenge or exposure to other defined stressors.

\section{ACKNOWLEDGMENTS}

We thank Sandy Braun, Willie Ford, and Beverly Horner for their technical assistance. This work was supported by the Veterans Administration National Center for Posttraumatic Stress Disorder and the Mental Health CRC at the University of California, San Diego (PHS MH30914-14).

\section{REFERENCES}

Allen R, Boublik J, Hauger R, Scott H, Rivier J, Brown M (1991): Neuropeptide Y radio-immunoassay: Characterization and application. Clin Exp Pharmacol Physiol 18: 825-833

Archelos J, Xiang J, Reinecke M, Lang R (1987): Regulation of release and function of neuropeptides in the heart. Cardiovascular Pharmacol 12:S45-S50

Boulenger J-P, Jerabek I, Jolicoeur F, Lavallee Y-J, Leduc R, Cadieux A (1996): Elevated plasma levels of neuropeptide $\mathrm{Y}$ in patients with panic disorder. Am J Psychiatr 153:114-116

Charney D, Woods S, Krystal J, Nagy L, Heninger G (1992): Noradrenergic neuronal dysregulation in panic disorder: The effects of intravenous yohimbine and clonidine in panic disorder patients. Acta Psychiatr Scand 86:273282

Charney D, Heninger G, Greier A (1984): Noradrenergic function in panic anxiety: Effects of yohimbine in healthy subjects and patients with agoraphobia and panic disorder. Arch Gen Psychiatr 41:751-763

Charney D, Heninger G, Sternberg D (1982): Assessment of $\alpha_{2}$-adrenergic autoreceptor function in humans: Effects of oral yohimbine. Life Sci 30:2033-2041

Colmers W, Bleakman D (1994): Effects of neuropeptide Y on the electrical properties of neurons. TINS 17:373-379

Dahlof P, Tarizzo V, Lundberg J, Dahlof C (1991): Alpha-and beta-adrenoceptor-mediated effects on nerve stimulation-evoked release of neuropeptide Y (NPY)-like immunoreactivity in the pithed guinea pig. J Autonomic Nerv System 35:199-210

Edvinsson L, Ekman R, Hedner P, Valdemarsson S (1990): 
Congestive heart failure: Involvement of perivascular peptides reflecting activity in sympathetic, parasympathetic and afferent fibres. Eur J Clin Invest 20:85-89

Goldberg M, Robertson D (1983); Yohimbine: A pharmacological probe for study of the $\alpha_{2}$-adrenoceptor. Pharmacol Rev 35:143-180

Gurguis G, Uhde T (1990): Plasma 3-methoxy-4-hydroxyphenethylene glycol (MHPG) and growth hormone responses to yohimbine in panic disorder patients and normal controls. Psychoneuroendocrinology 15:217-224

Haass M, Cheng B, Richardt G, Lang R, Schomig A (1989): Characterization and presynaptic modulation of stimulation-evoked exocytotic co-release of noradrenaline and neuropeptide $Y$ in guinea pig heart. NaunynSchmiedebergs Arch Pharmacol 339:71-78

Hedner T, Edgar B, Edvinsson L, Hedner J, Persson B, Pettersson A (1992): Yohimbine pharmacokinetics and interaction with the sympathetic nervous system in normal volunteers. Eur J Clin Pharmacol 43:651-666

Heilig M, McLeod S, Brot M, Heinrichs S, Menzaghi F, Koob G, Britton K (1993): Anxiolytic-like action of neuropeptide Y: Mediation by Y1 receptors in amygdala, and dissociation from food intake effects. Neuropsychopharmacology 8:357-363

Heilig M, Widerlov E (1990): Neuropeptide Y: An overview of central distribution, functional aspects, and possible involvement in neuropsychiatric illnesses. Acta Psychiatr Scand 82:95-114

Heilig M, Soderpalm B, Enger J, Widerlov E (1989): Centrally administered neuropeptide Y (NPY) produces anxiolyticlike effects in animal anxiety models. Psychopharmacology 98:524-529

Henaur S, Gillespie H, Hollister L (1984): Yohimbine and the model anxiety state. J Clin Psychiatr 45:512-515

Holmberg G, Gershon S, Beck L (1962): Yohimbine as an autonomic test drug. Nature 193:1313-1314

Illes P, Regenold J (1990): Interaction between neuropeptide $\mathrm{Y}$ and noradrenaline on central catecholamine neurons. Nature 344:62-63
Lundberg J, Rudehill A, Sollevi A (1989): Pharmacological characterization of neuropeptide $\mathrm{Y}$ and noradrenaline mechanisms in sympathetic control of pig spleen. Eur J Pharmacol 163:103-113

Pernow J, Lundberg J (1989): Modulation of noradrenaline and neuropeptide $\mathrm{Y}$ (NPY) release in the pig kidney in vivo: Involvement of alpha 2, NPY and angiotensin II receptors. Naunyn-Schmiedebergs Arch Pharmacol 340: 379-385

Pernow J (1988): Co-release and functional interactions of neuropeptide $\mathrm{Y}$ and noradrenaline in peripheral sympathetic vascular control. Acta Physiologica Scand 568: (Suppl)1-56

Southwick S, Krylstal J, Morgan C, Johnson D, Nagy L, Nicolaou A, Heninger G, Charney D (1993): Abnormal noradrenergic function in posttraumatic stress disorder. Arch Gen Psychiatr 50:266-274

Stein M, Hauger R, Dhalla K, Chartier M, Asmundson G (1996): Plasma neuropeptide $\mathrm{Y}$ in anxiety disorders: Findings in panic disorder and social phobia. Psychiatry Res 59:183-188

Travernier G, Damase-Michel C, Portolan G, Tran M, Montastruc J (1992): Is neuropeptide $Y$ coreleased with catecholamines in experimental arterial hypertension following sinoaortic denervation? Naunyn-Schmiedebergs Arch Pharmacol 345:431-436

Wahlestedt C, Reis D (1993): Neuropeptide Y-related peptides and their receptors-are the receptors potential therapeutic drug targets? Annu Rev Pharmacol Toxicol 32:309-352

Wahlestedt C, Pich E, Koob G, Yee F, Heilig M (1993): Modulation of anxiety and neuropeptide Y-Y1 receptors by antisense oligodeoxynucleotides. Science 259:528-531

Widerlov E, Heilig M, Ekman R, Wahlestedt C (1989): Neuropeptide $\mathrm{Y}-$ possible involvement in depression and anxiety. In Mutt V, Fuxe K, Hokfelt T, Lundberg JM (eds), Nobel Conference on NPY. New York, Raven Press, pp 331-342 